\title{
Application of Nitrogenated Holey Graphene for Detection of Volatile Organic Biomarkers in Exhaled Breath of Humans With Chronic Kidney Disease: A Density Functional Theory Study
}

Roya Majidi ( $\square$ royamajidi@gmail.com )

Shahid Rajaee Teacher Training University https://orcid.org/0000-0001-8451-3695

Marzieh Nadafan

Shahid Rajaee Teacher Training University

\section{Research Article}

Keywords: Nitrogenated holey graphene, chronic kidney disease, biosensor, energy band gap, density functional theory

Posted Date: June 7th, 2021

DOI: https://doi.org/10.21203/rs.3.rs-569980/v1

License: (a) (i) This work is licensed under a Creative Commons Attribution 4.0 International License.

Read Full License 


\section{Abstract}

The possibility of using nitrogenated holey graphene (NHG) sheet to detect volatile organic biomarkers in exhaled breath of humans with kidney disease is investigated. Heptanal, hexanal, pentanal, and isoperene are known as the prominent biomarkers of chronic kidney disease. Adsorption of these molecules on NHG sheet is studied using density functional theory. All the molecules are weakly physisorbed on NHG sheet, which predicts easy desorption and the possibility of using NHG sheet as a reusable sensor. The NHG sheet acts as a semiconductor with a direct band gap. Adsorption of the considered molecules causes n-type semiconducting properties in the sheet. Increasing the concentration of the adsorbed molecules decreased the energy band gaps and consequently increased the electric conductivity of NHG sheet. Hence, the electronic properties of NHG sheet are sensitive to the presence and concentration of heptanal, hexanal, pentanal, and isoperene molecules. Our results open a new opportunity to design a new sensor to diagnose chronic kidney disease using exhaled breath analysis.

\section{Introduction}

Chronic kidney disease (CKD) is known as a global public health problem [1]. The number of patients suffering from CKD is significantly growing worldwide. Early treatment can prevent or delay complications of decreased kidney function, slow the progression of kidney disease, and reduce the risk of cardiovascular disease [1, 2]. Unfortunately, most of patients are diagnosed at a late stage because the initial stage of CKD usually is clinically silent and asymptomatic. Thus, exploration of rapid, safe, and inexpensive methods for early unambiguous diagnosis of renal insufficiency is vital. Nowadays, the possibility of using the exhaled breath analysis as a non-invasive method for early diagnosis of progressive disease and response to therapeutic interventions in different illnesses has been investigated [3-12]. In this context, the impact of CKD on the composition of the exhaled breath in humans and experimental animals several studies has been explored $[2,12]$. It is found that CKD significantly changes the composition of exhaled breath [12]. Monitoring the concentration of over fifty gases in the exhaled breath of patients with CKD reveals that heptanal, hexanal, pentanal, and isoperene are the prominent biomarkers of CKD $[2,12,13]$.

The past several decades have witnessed increasing attention by researchers to nanomaterials owing to some superior properties such as high stability, small size, high specific surface area, suitable surface modification property, and adjustable lifetime [14-16]. Nanomaterials have been the interest of many theoretical studies and became an exciting area for experimentalists [14-39]. Among various nanomaterials, carbon-based ones especially carbon nanotubes, graphene, and graphyne have demonstrated their appropriateness for wide spectrum of applications ranging from electronics to optics, sensors, and biodevices [17, 18-21]. One of the most promising applications is gas adsorption and sensing. The sensing performance of carbon nanotubes, graphene, and graphyne towards $\mathrm{NO}_{2}, \mathrm{NH}_{3}, \mathrm{H}_{2} \mathrm{O}$, $\mathrm{CO}, \mathrm{H}_{2} \mathrm{~S}, \mathrm{SO}_{2}$, etc. have been studied extensively [21-26]. The results showed that the sensors based on carbon nanotubes, graphene and its derivatives are capable for detecting individual molecules with a relatively high sensitivity. Furthermore, these materials are suggested as highly sensitive biosensors to 
detect the expression of typical biological molecules at early stage of cancer [27-29]. In terms of the application of nanomaterials as sensors, sheets and nanotubes based on phosphorene, silicene, germanene, antimonene, and arsenene are also receiving great attentions from both the academic and industrial communities [30-34]. The findings reported that different gas molecules could be sensed through adsorption on these materials. Motivated by these results, researchers continue to seek for novel sensitive sensors based on nanomaterials.

Two-dimensional crystals with $\mathrm{C}_{2} \mathrm{~N}$ stoichiometry named nitrogenated holey graphene (NHG) have recently been synthesized [35-38]. This two-dimensional material, unlike pristine graphene which lacks a band gap and needs to be engineered to open the gap for practical application, has a direct band gap [35-39]. It could have applications similar to or even more fascinating than graphene. In this study, we have investigated the structural and electronic properties of NHG when adsorbing gas molecules using density functional theory (DFT).

\section{Computational Details}

All DFT calculations are performed with the OpenMX3.8 code [40]. The Perdew, Burke, and Ernzerhof (PBE) approach from the generalized gradient approximation (PBE-GGA) is applied to describe the exchange-correlation functional [41]. The tractable norm-conserving pseudopotential proposed by Morrison, Bylander, and Kleinman [42], which is a norm-conserving version of the ultrasoft pseudopotential by Vanderbily [43] is used. The empirical correction method described by Grimme (DFTD2) is used to describe the van der Waals (vdW) interactions [44]. The energy cutoff is adjusted to 100 Ry. The pseudoatomic orbitals (PAO) basis functions are specified by s2p2d1 for $\mathrm{C}, \mathrm{N}, \mathrm{O}$, and $\mathrm{H}$ atoms with the cutoff radii of the basis functions set to the value of seven. Atomic coordinates are fully optimized until the forces on each of the constituent atoms are converged to $0.05 \mathrm{eV} / \AA . \AA$. The periodic boundary conditions are applied in all dimensions. A vacuum space of $25 \AA$ is left in the perpendicular direction to the NHG sheet to prevent the columbic interaction between the neighboring slabs. Mulliken population analysis is performed to obtain the charges on individual atoms [45].

In the present work, adsorption of heptanal, hexanal, pentanal, and isoperene molecules are investigated. Atomic structures of heptanal $\left(\mathrm{C}_{7} \mathrm{H}_{14} \mathrm{O}\right)$, hexanal $\left(\mathrm{C}_{6} \mathrm{H}_{12} \mathrm{O}\right)$, pentanal $\left(\mathrm{C}_{5} \mathrm{H}_{10} \mathrm{O}\right)$, and isoprene $\left(\mathrm{C}_{5} \mathrm{H}_{8}\right)$ are shown in Fig. 1.

In order to evaluate the stability of adsorption of the configurations, the adsorption energy is defined as,

$E_{\text {ads }}=\left(E_{\text {sheet }+ \text { molecule }}-E_{\text {sheet }}-E_{\text {molecule }}\right)$

here, $\mathrm{E}_{\text {molecule, }} \mathrm{E}_{\text {sheet, }}$ and $\mathrm{E}_{\text {sheet+molecule }}$ are the total energies of the molecule, NHG sheet, and NHG sheet with adsorbed molecule, respectively. With such definition, negative adsorption energy indicated that the adsorption is exothermic. The nudged elastic band (NEB) method [46] is employed to search for the optimal adsorption distance. 
To study the electronic properties of, the electronic band structures and density of states (DOS) are calculated. Along each high symmetry line in the Brillouin zone, $21 \mathrm{~K}$-points are considered. The electric conductivity of the systems, , is estimated by

$\sigma \infty \mathrm{e}^{-\mathrm{Eg} / \mathrm{K}_{\mathrm{B}} T}$

Here, $\mathrm{E}_{\mathrm{g}}$ is the band gap of the system. $\mathrm{K}_{\mathrm{B}}$ and $\mathrm{T}$ denote the Boltzmann's Constant and temperature, respectively. The electric conductivity is obtained at room temperature.

\section{Results And Discussion}

Initially, the structural and electronic properties of NHG are studied. To model a monolayer of NHG sheet, a $2 \times 2$ supercell is considered as shown in Fig. 2(a). The primitive unit cell of $\mathrm{NHG}$ with $\mathrm{C}_{2} \mathrm{~N}$ stoichiometry contains $12 \mathrm{C}$ and $6 \mathrm{~N}$ atoms and its lattice constant is $8.33 \AA$. The fully relaxed $\mathrm{C}-\mathrm{N}$ bond length is calculated to be $1.34 \AA$. The $\mathrm{C}-\mathrm{C}$ bonds have two different values. The $\mathrm{C}-\mathrm{C}(1)$ bond at the border of the hole is $1.48 \AA$ and the $\mathrm{C}-\mathrm{C}(2)$ bond shared between benzene and pyrazine rings is $1.41 \AA$. The results are in good agreement with those reported in the previous reports [47]. The electron density difference of NHG sheet is illustrated in Fig. 2(b). Here, the yellow color regions indicate electron accumulation, while the cyan regions indicate electron depletion. It is clear that the charge density is mainly distributed between $\mathrm{C}$ and $\mathrm{N}$. This suggests that the covalent bond is formed between $\mathrm{C}$ and $\mathrm{N}$. The holey site is surrounded by negatively charged $\mathrm{N}$ atoms. The results are in good agreement with previous studied [48-49]. Mulliken atomic populations indicate that each $\mathrm{C}$ atom donates $0.22 \mathrm{e}$, and each $\mathrm{N}$ atom gains $0.44 \mathrm{e}$.

The electronic band structure and DOS of NHG sheet are illustrated in Fig. 3. There is a band gap of 1.82 $\mathrm{eV}$ between top of the valence band and bottom of the conduction band at $\Gamma$ point of the Brilliouin zone. Hence, NHG is a semiconductor with a direct band gap. It is in accordance with prior studies which predicted a band gap of $1.60,1.66,1.70,1.82$ and $2.47 \mathrm{eV}$ based on DFT calculations, and optically measured a band gap of $1.96 \mathrm{eV}$ [35-39].

To find the most stable adsorption configuration, the gas molecules are placed at all possible adsorption sites on the sheet. The possible adsorption sites on NHG are top of $\mathrm{C}$ and $\mathrm{N}$ atoms, the center of $\mathrm{C}-\mathrm{N}$ and $\mathrm{C}-\mathrm{C}$ bond, the center of benzene and pyrazine rings, and hollow site. For each adsorption site, different molecular orientations are examined. The center of hollow site is found to be the most favorable site for heptanal, hexanal, and pentanal adsorption. The adsorption behavior of isoperene on NHG sheet is different from other molecules. Isoperene is preferably adsorbed on top of the pyrazine ring. As an example, NHG sheet with adsorbed heptanal and isoperene molecules are shown in Fig. 4 (a and b). It is found that there is no structural deformation in NHG sheets after adsorption of the molecules, further indicating that the stability of NHG sheet. An energy scan of the adsorbed molecules at the most stable adsorption sites is performed to obtain the optimal adsorption distance of the molecules on NHG sheet. Adsorption distance is defined as the shortest atom-to-sheet distance between the molecule and NHG sheet. 
The NEB is also used to analyze the change of total energy as a function of the adsorption distance. As an example, the change of total energy as a function of the adsorption distance for adsorption of heptanal, hexanal, pentanal, and isoperene on NHG sheet is shown in Fig. 5. The adsorption energies and distances of the most favorable structures are summarized in Table 1. A negative value of adsorption energy indicated that the adsorption is exothermic. The greater absolute value of adsorption energy means the more stable system. The binding strength follows the trend: pentanal < hexanal $<$ heptanal < isoperene. The considered molecules prefer to physically adsorb on NHG sheet with small adsorption energies.

Table 1

Adsorption energy, $E_{\mathrm{ads}}$, and adsorption distance, $d$.

\begin{tabular}{|lllll|}
\hline & heptanal & hexanal & pentanal & isoperene \\
\hline$E_{\text {ads }}(\mathrm{eV})$ & -0.30 & -0.19 & -0.17 & -0.35 \\
\hline$d(\AA)$ & 2.6 & 2.7 & 2.9 & 2.6 \\
\hline
\end{tabular}

In order to gain further insight about the interlayer interactions, the charge densities are calculated. As an example, the total electron density and electron density difference of NHG sheet with adsorbed heptanal are shown in Fig. 6. Here, no electron orbital overlap and electron accumulation between adsorbed molecule and NHG sheet are observed. This feature indicates there are no chemical bonds the molecules and NHG sheet, indicating that the systems are trend to physical adsorption. Mulliken charge analysis shows that the adsorbed molecules act as charge acceptors. Each adsorbed heptanal, hexanal, pentanal, and isoperene gain $0.012,0.011,0.10$, and 0.059 e from NHG sheet, respectively. The little charge transfers as well as small adsorption energies confirm the physical adsorption of the considered molecules on NHG sheet.

The electronic band structure and DOS are studied for the most stable structures of each molecule adsorbed on NHG sheet (Fig. 7). As shown, flat occupied states and sharp DOS peaks are appeared in the band gap at energy of about $-0.65,-0.64,-0.62$, and $-0.68 \mathrm{eV}$ for heptanal, hexanal, pentanal, and isoperene, respectively. It means that NHG sheets in the presence of the considered molecules are n-type semiconductors. The energy band gap is defined as the difference between the highest occupied and lowest unoccupied states. The band gaps are 1.30, 1.25, 1.25, and $1.39 \mathrm{eV}$ after adsorption of heptanal, hexanal, pentanal, and isoperene molecules, respectively (Table 2). 
Table 2

Energy band gap, $E_{g}$, and conductivity, $\sigma$, for $n=1-4$ adsorbed molecules.

\begin{tabular}{|c|c|c|c|c|c|}
\hline & $n$ & heptanal & hexanal & pentanal & isoperene \\
\hline \multirow[t]{4}{*}{$E_{\mathrm{g}}(\mathrm{eV})$} & 1 & 1.30 & 1.25 & 1.25 & 1.39 \\
\hline & 2 & 1.16 & 1.13 & 1.16 & 1.25 \\
\hline & 3 & 1.13 & 1.07 & 1.0 & 1.25 \\
\hline & 4 & 1.12 & 0.92 & 0.91 & 1.24 \\
\hline \multirow[t]{4}{*}{$\sigma$} & 1 & $1.1 \times 10^{-11}$ & $3.0 \times 10^{-11}$ & $3.0 \times 10^{-11}$ & $2.0 \times 10^{-12}$ \\
\hline & 2 & $1.7 \times 10^{-10}$ & $3.1 \times 10^{-10}$ & $1.7 \times 10^{-10}$ & $3.0 \times 10^{-11}$ \\
\hline & 3 & $3.1 \times 10^{-10}$ & $9.9 \times 10^{-10}$ & $3.8 \times 10^{-9}$ & $3.0 \times 10^{-11}$ \\
\hline & 4 & $3.7 \times 10^{-10}$ & $1.8 \times 10^{-8}$ & $2.2 \times 10^{-8}$ & $3.7 \times 10^{-11}$ \\
\hline
\end{tabular}

The electric conductivity of NHG sheet at $300 \mathrm{~K}$ is calculated to be $4.8 \times 10^{-16}$. The electric conductivity of NHG sheet after adsorption of the molecules is listed in Table 2. It is found that the electric conductivity of NHG sheet in the presence of the adsorbed molecules is more than that of pure NHG sheet.

To study the sensitivity of the electronic properties of NHG to the concentration of the considered molecules, the number of adsorbed molecules is changed from one to four. As an example, atomic structure NHG sheet in the presence of four hexanal molecules is shown in Fig. 4 (c) and the electronic band structure and DOS of NHG in the presence of four pentanal molecules are shown in Fig. 8. As shown, the numbers of occupied states and sharp DOS peaks below the Fermi level are increased by increasing the number of adsorbed molecules. The energy band gap and electric conductivity as a function of the number of adsorbed molecule is listed in Table 2. Increasing the concentration of the adsorbed molecules decreased the energy band gap and consequently increased the electric conductivity (Table 2).

\section{Conclusions}

Heptanal, hexanal, pentanal, and isoperene molecules are the prominent biomarkers of CKD. Motivated by the recent realization of two-dimensional nanomaterials as gas sensors, we have investigated the adsorption of these molecules on NHG sheet. Adsorption energies, adsorption distance, electronic band structures, density of states, energy band gap, and electric conductivity are calculated using density functional theory calculations. The large adsorption distances and small indicate these molecules are energetically favorable to physically adsorb on NHG sheet. The NHG sheet with intrinsic semiconducting properties exhibit n-type semiconducting properties after adsorption of the considered molecules. It is found that the energy band gap of NHG sheet is sensitive to the concentration of the adsorbed molecules. 
Increasing the number of the adsorbed molecules decreased the energy band gap and consequently increased the conductivity. Our results suggested that NHG sheet could be used as a proper gas sensor for detection of heptanal, hexanal, pentanal, and isoperene as the prominent biomarkers to diagnose CKD.

\section{Declarations}

\section{Acknowledgement}

The work was supported by Shahid Rajaee Teacher Training University.

\section{References}

1. S. Levey, R. Atkins, J. Coresh, E.P. Cohen, A.J. Collins, K-U. Eckardt, M.E. Nahas, B.L. Jaber, M. Jadoul, A. Levin, N.R. Powe, J. Rossert, D.C. Wheeler, N. Lameire, G. Eknoyan, Chronic kidney disease as a global public health problem: Approaches and Initiatives - A position statement from kidney disease improving global outcomes, Kidney Int. 72 (2007) 247-259.

2. Grabowska-Polanowska, J. Faber, M. Skowron, P. Miark, A. Pietrzyck, I.S. liwka, A. Amannd, Detection of potential chronic kidney disease markers in breath using gas chromatography with mass-spectral detection coupled with thermal desorption method, J. Chromatogr, A 1301 (2013) 179-189.

3. Amann, P. Spanel, D. Smith, Breath analysis: the approach towards clinical applications, Mini Rev. Med. Chem. 7 (2007) 115-129.

4. Hakim, Y.Y. Broza, O. Barash, N. Peled, M. Phillips, A. Amann, H. Haick, Volatile organic compounds of lung cancer and possible biochemical pathways, Chem. Rev. 112 (2012) 5949-5966.

5. Majidi, M. Nadafan, Detection of exhaled gas by $\mathrm{Y}$-graphyne and twin-graphene for early diagnosis of lung cancer: Adensity functional theory study, J. Phys. Lett. A 384 (2020) 126036.

6. J. Mazzone, J. Hammel, R. Dweik, et al., Diagnosis of lung cancer by the analysis of exhaled breath with a colorimetric sensor array, Thorax 62 (2007) 565-568.

7. Buszewski, M. Kesy, T. Ligor, A. Amann, Human exhaled air analytics: biomarkers of diseases, Biomed. Chromatogr. 21 (2007) 553-566.

8. Ligor, M. Ligor, A. Amann, C. Ager, M. Bachler, A. Dzien , B. Buszewski, The analysis of healthy volunteers' exhaled breath by the use of solid-phase microextraction and GC-MS, J. Breath Res. 2 (2008) 046006.

9. Capuano, A. Catini, R. Paolesse, C. Di Natale, Sensors for lung cancer diagnosis, J. Clin. Med. 8 (2019) 235.

10. Behera, R. Joshi, G.K. Anil Vishnu, S. Bhalerao, H.J. Pandya, Electronic nose: a non-invasive technology for breath analysis of diabetes and lung cancer patients, J. Breath Res. 2 (2019) 024001.

11. C. Sruthy, V. Nagarajan, R. Chandiramouli, Interaction studies of kidney biomarker volatiles on black phosphorene nanoring: A first-principles investigation, J. Mol. Graph. Model. 97 (2020) 107566. 
12. Meinardi, K-B. Jin, B. Barletta, D.R. Blake, N.D. Vaziri, Exhaled breath and fecal volatile organic biomarkers of chronic kidney disease, BBA-Biomembranes 1830 (2013) 2531-2537.

13. Trovarelli, F. Brunori, G.E. De Medio, M. Timio, G. Lippi, M.A. Pelli, E. Capodicasa, Onset, time course, and persistence of increased haemodialysis-induced breath isoprene emission, Nephron 88 (2000) 44-47.

14. Hibino, H. Kageshima, M. Kotsugi, F. Maeda, F.-Z. Guo, Y. Watanabe, Dependence of electronic properties of epitaxial few-layer graphene on the number of layers investigated by photoelectron emission microscopy, Phys. Rev. B. 79 (2009) 125437.

15. Songa, J. Gaob, L. Wuc, Fluorouracil drug sensing characteristics of pristine and Al-doped BC3 nanosheets: Quantum chemical study, Comput. Theor. Chem. 1182 (2020) 112847.

16. Jeevanandam, A. Barhoum, Y.S. Chan, A. Dufresne, M.K. Danquah, Review on nanoparticles and nanostructured materials: history, sources, toxicity and regulations, Beilstein J. Nanotechnol. 9 (2018) 1050-1074.

17. Mortazavi, E.V. Podryabinkin, S. Roche, T. Rabczuk, X. Zhuang, A.V. Shapeev, Machine-learning interatomic potentials enable first-principles multiscale modeling of lattice thermal conductivity in graphene/borophene heterostructures, Mater. Horiz. 7 (2020) 2359.

18. Maiti, X. Tong, X. Mou, K. Yang, Carbon-based nanomaterials for biomedical applications: A Recent Study, Front Pharmacol. 9 (2018) 1401.

19. Notarianni, J. Liu, K. Vernon, N. Motta, Beilstein J. Nanotechnol. 7 (2016) 149-196. Synthesis and applications of carbon nanomaterials for energy generation and storage

20. Esmailpour, R. Majid, H.R. Taghiyari, M. Ganjkhani, S.M. Mohseni Armaki, A.N. Papadopoulos, Improving fire retardancy of beechwood by graphene, Polymers 12 (2020) 303.

21. D.N. Bezzon, T.L A. Montanheiro, B.R.C. de Menezes, R.G. Ribas,V.A.N. Righetti,K.F. Rodrigues, G.P. Thim, Carbon Nanostructure-based Sensors: A Brief Review on Recent Advances, Adv. Mater. Sci. Eng. 2019 (2019) 1-21.

22. You, J. Deng, X. Tan, N. Gorjizadeh, M. Yoshimura, S.C. Smith, V. Sahajwalla, R.K. Joshi, On the mechanism of gas adsorption for pristine, defective and functionalized graphene, Phys. Chem. Chem. Phys. 19 (2017) 6051-6056.

23. Majidi, A.R. Karami, Caffeine and nicotine adsorption on perfect, defective and porous graphene sheets, Diam. Relat. Mater. 66 (2016) 47-51.

24. Deb, D. Paul, D. Pegu, U. Sarkar, Adsorption of hydrazoic acid on pristine graphyne sheet: A computational study, Wuli Huaxue Xuebao, Acta Physico - Chimica Sinica 34 (2018) 537-542.

25. V. Nagarajan, S. Dharani, R. Chandiramouli, Density functional studies on the binding of methanol and ethanol molecules to graphyne nanosheet, Comp. Theor. Chem. 1125 (2018) 86-94.

26. R. Karami, R. Majidi, Detection of toxic gases with graphyne nanotubes: A density functional theory study, Chem. Lett. 44 (2015) 1071-1073. 
27. Majidi, U. Sarkar, Detection of $\mathrm{NO}_{x}$ and $\mathrm{CO}_{x}(x=1,2)$ molecules with $\mathrm{T} 4,4,4$-graphyne: a density functional theory study, Mol. Phys. 46 (2020) 1383-1389.

28. Q. Wan, Y. Xu, H. Xiao, Exhaled gas detection by Ir-doped CNT for primary diagnosis of lung cancer, AlP Adv. 8 (2018) 105128.

29. Q. Wan, Y. Xu, X. Zhang, Adsorption Properties of Typical Lung Cancer Breath Gases on Ni-SWCNTs through Density Functional Theory, J. Sensors 2017 (2017) (1-8).

30. R. Majidi, R. Ramazani, Detection of $\mathrm{HF}$ and $\mathrm{H}_{2} \mathrm{~S}$ with pristine and Ti-embedded twin graphene: $\mathrm{A}$ density functional theory study, J. Phys. Chem. Solids 132 (2019) 31.

31. Prasongkit, R.G. Amorim, S. Chakraborty, R. Ahuja, R.H. Scheicher, V. Amornkitbamrung, Highly sensitive and selective gas detection based on silicene, . Phys. Chem. C 119 (2015) 16934-16940.

32. Abbasi, Adsorption of ozone molecules on AlP-codoped stanene nanosheet: A density functional theory study, J. Nanoanalysis., 6 (2019) 60-71.

33. Song, J. Gaob, L. Wu, Fluorouracil drug sensing characteristics of pristine and Al-doped BC3 nanosheets: Quantum chemical study, Comput. Theor. Chem. 1182 (2020) 112847.

34. Srivastava, Abhishek, N.K. Jaiswal, First-principles investigation of $\mathrm{CO}_{2}$ and $\mathrm{NH}_{3}$ adsorption on antimonene nanoribbons, Mater. Today: Proceedings: 28 (2020) 65-69.

35. Mahmood, E. Kwang Lee, M. Jung, et al., Nitrogenated holey two-dimensional structures, Nat. Commun. 6 (2015) 6486.

36. M. Tromer, M.G.E. da Luz, M.S. Ferreira, L. Felipe, C. Pereira, Atomic adsorption on nitrogenated holey graphene, J. Phys. Chem. C 121 (2017) 3055-3061.

37. Yagmurcukardes, S. Horzum, E. Torun, F.M. Peeters, R. Tugrul Senge, Nitrogenated, phosphorated and arsenicated monolayer holey graphenes, Phys. Chem. Chem. Phys. 18 (2016) 3144-3150.

38. V.R. Nulakani, V. Subramanian, A theoretical study on the design, structure, and electronic properties of novel forms of graphynes, J. Phys. Chem. C 120 (2016) 15153-15161.

39. majidi. M. Odelius, Sh. AlTaha, Structural and electronic properties of nitrogenated holey nanotubes: A density functional theory study, Diam. Relat. Mater. 82 (2018) 96-101.

40. Ozaki, H. Kino, J. Yu, M.J. Han, N. Kobayashi, M. Ohfuti, F. Ishii, F., et al., User's Manual of OpenMX Version 3.8, http://www.openmx-square.org.

41. P. Perdew, K. Burke, M. Ernzerhof, Generalized gradient approximation made simple, Phys. Rev. Lett. 77 (1996) 3865.

42. Morrison, D.M. Bylander, L. Kleinman, Nonlocal hermitian norm-conserving vanderbilt pseudopotential, Phys. Rev. B 47 (1993) 6728.

43. Vanderbilt, Soft self-consistent pseudopotentials in a generalized eigenvalue formalism, Phys. Rev. B 41 (1990) 7892.

44. J. Grimme, Semiempirical GGA-type density functional constructed with a long-range dispersion correction, Comput. Chem. 27 (2006) 1787-1799. 
45. S. Mulliken, Electronic population analysis on LCAO-MO molecular wave functions. IV. Bonding and antibonding in LCAO and valence-Bond theories, J. Chem. Phys. 23 (1955) 2343-2346.

46. Henkelman and $\mathrm{H}$. Jonsson, Improved tangent estimate in the nudged elastic band method for finding minimum energy paths and saddle points, J. Chem. Phys. 113 (2000) 9978.

47. Huang, H-H. Wu, C. Chi, J. Zhu, B. Huang, T-Y. Zhang, Out-of-plane ion transport makes nitrogenated holey graphite a promising high-rate anode for both $\mathrm{Li}$ and $\mathrm{Na}$ ion batteries, Nanoscale 11 (2019) 18758-18768.

48. Y. Xu, H.K. Dong, L.B. Shi, First principles investigation of nitrogenated holey graphene, Physica E 98 (2018) 135-139.

49. Sahin, Structural and phononic characteristics of nitrogenated holey graphene Phys. Rev. B 92 (2015) 085421.

\section{Figures}




\section{heptanal}

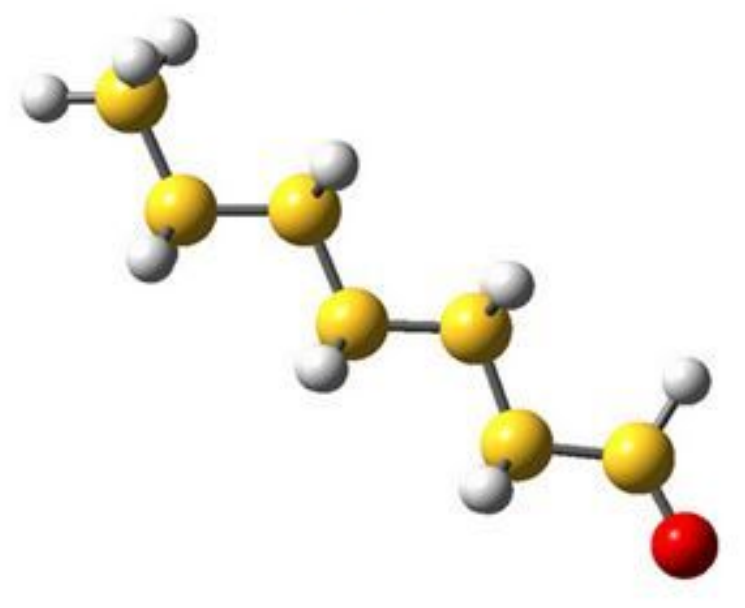

pentanal

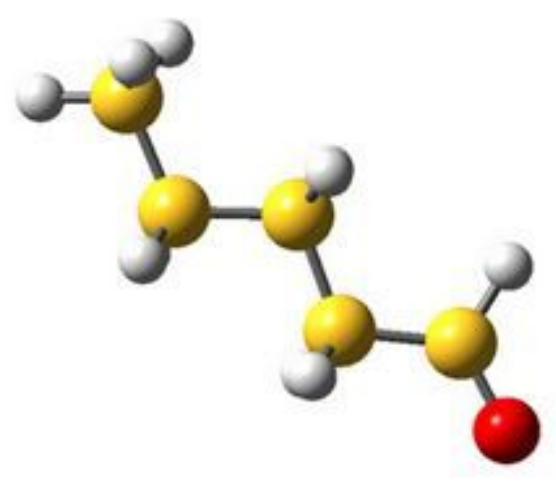

$\mathrm{C}$

\section{hexanal}

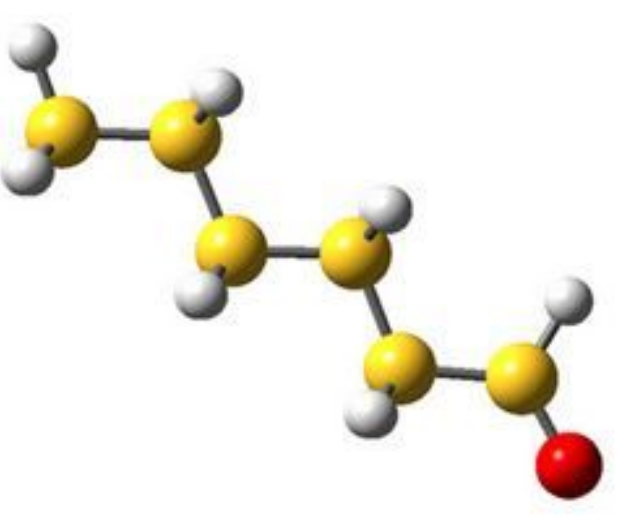

isoprene

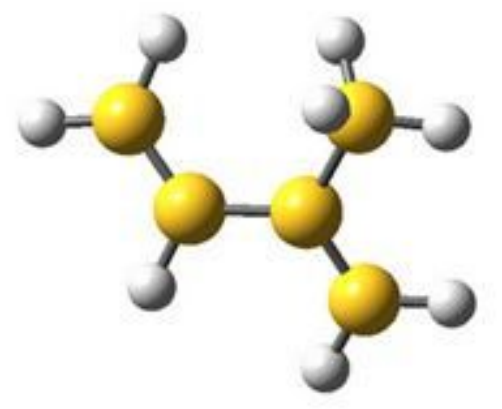

- $\mathrm{O}, \mathrm{H}$

Figure 1

Atomic structures of heptanal (C7H140), hexanal (C6H12O), pentanal (C5H100), and isoprene (C5H8). 

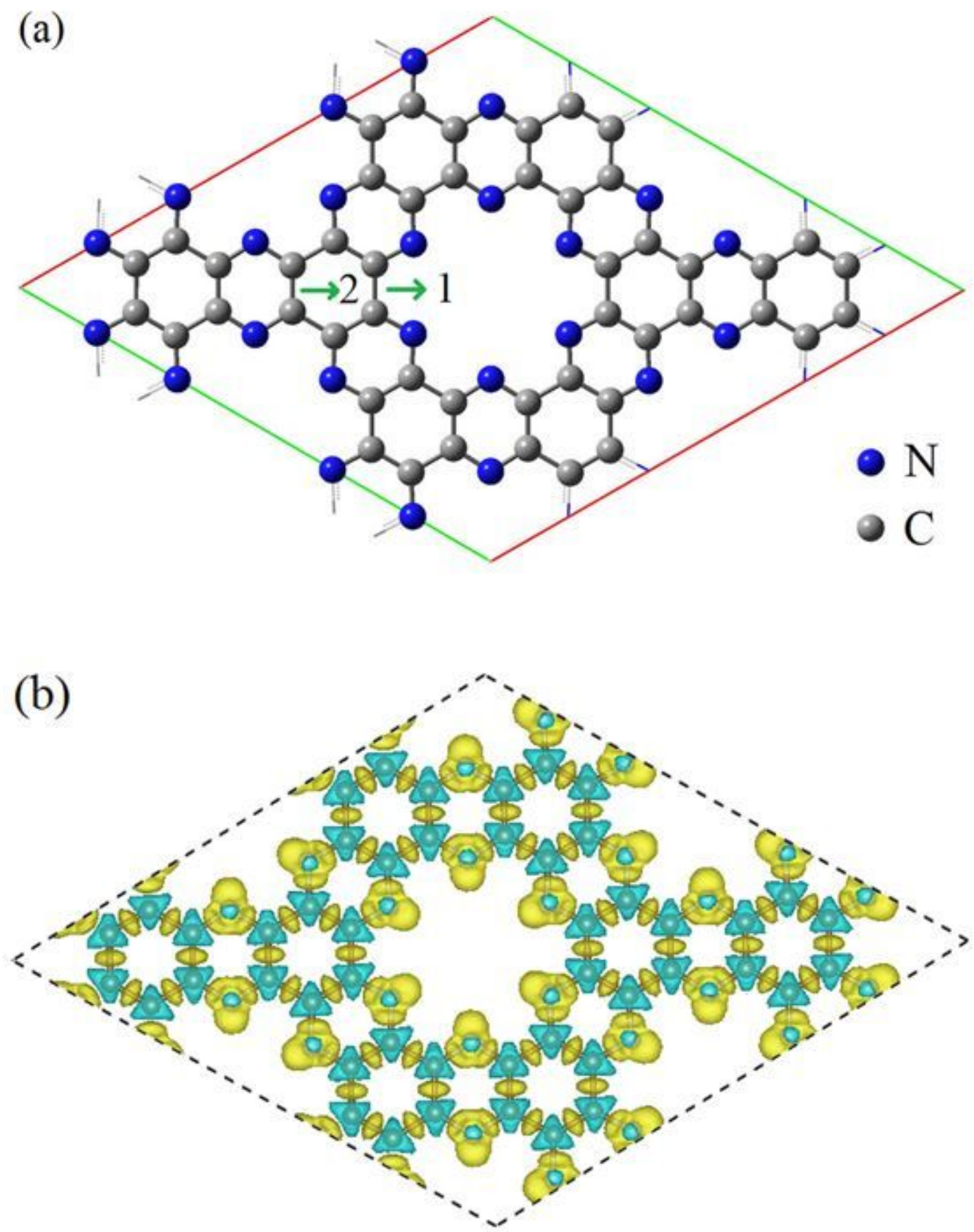

Figure 2

(a) Atomic structure and (b) electron density difference of NHG sheet. (The value of isosurface is $0.013 \mathrm{e} /$ Å3. The yellow and cyan regions show the gain and loss of electron, respectively.) 


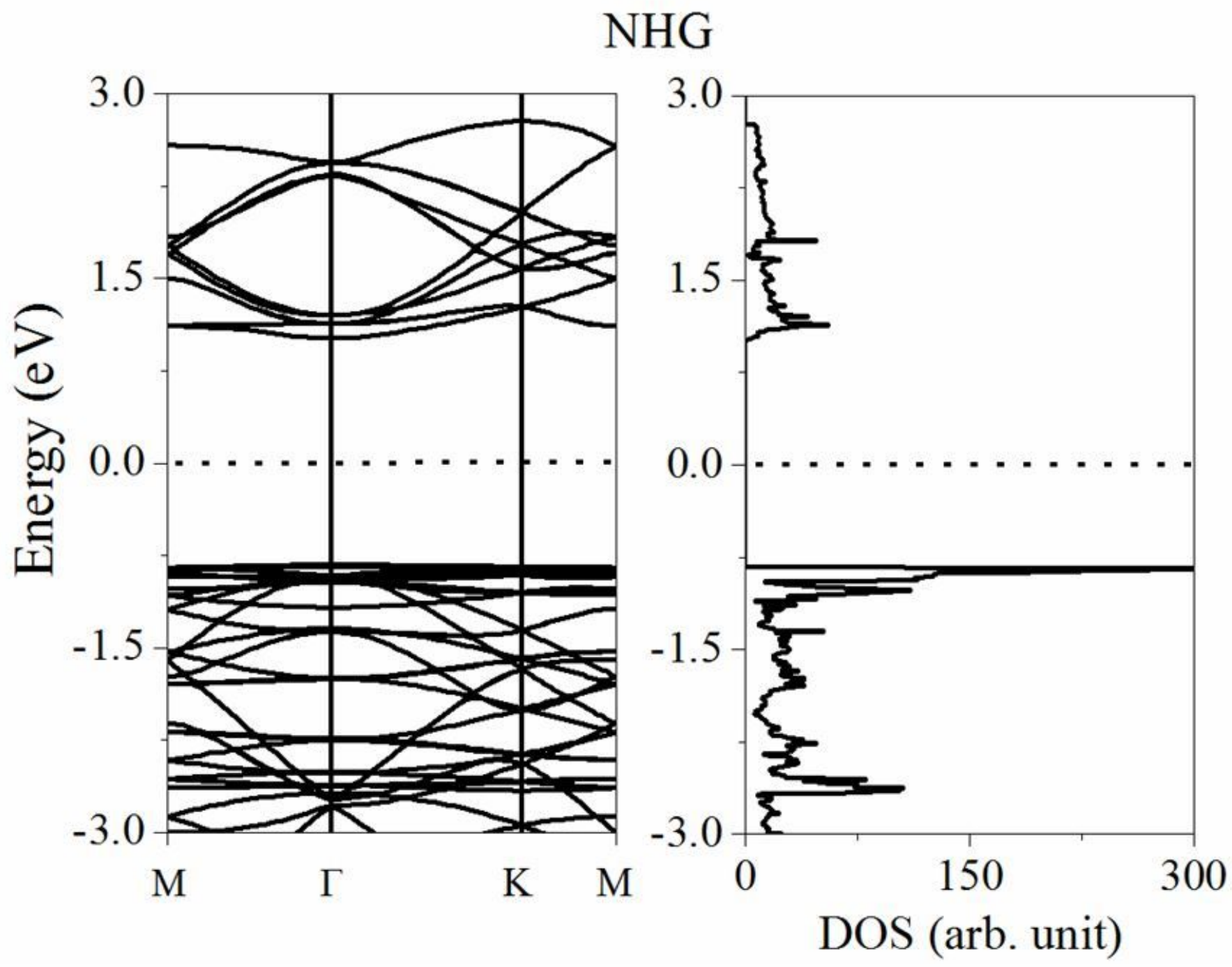

Figure 3

Electronic band structures and DOS of NHG sheet. 

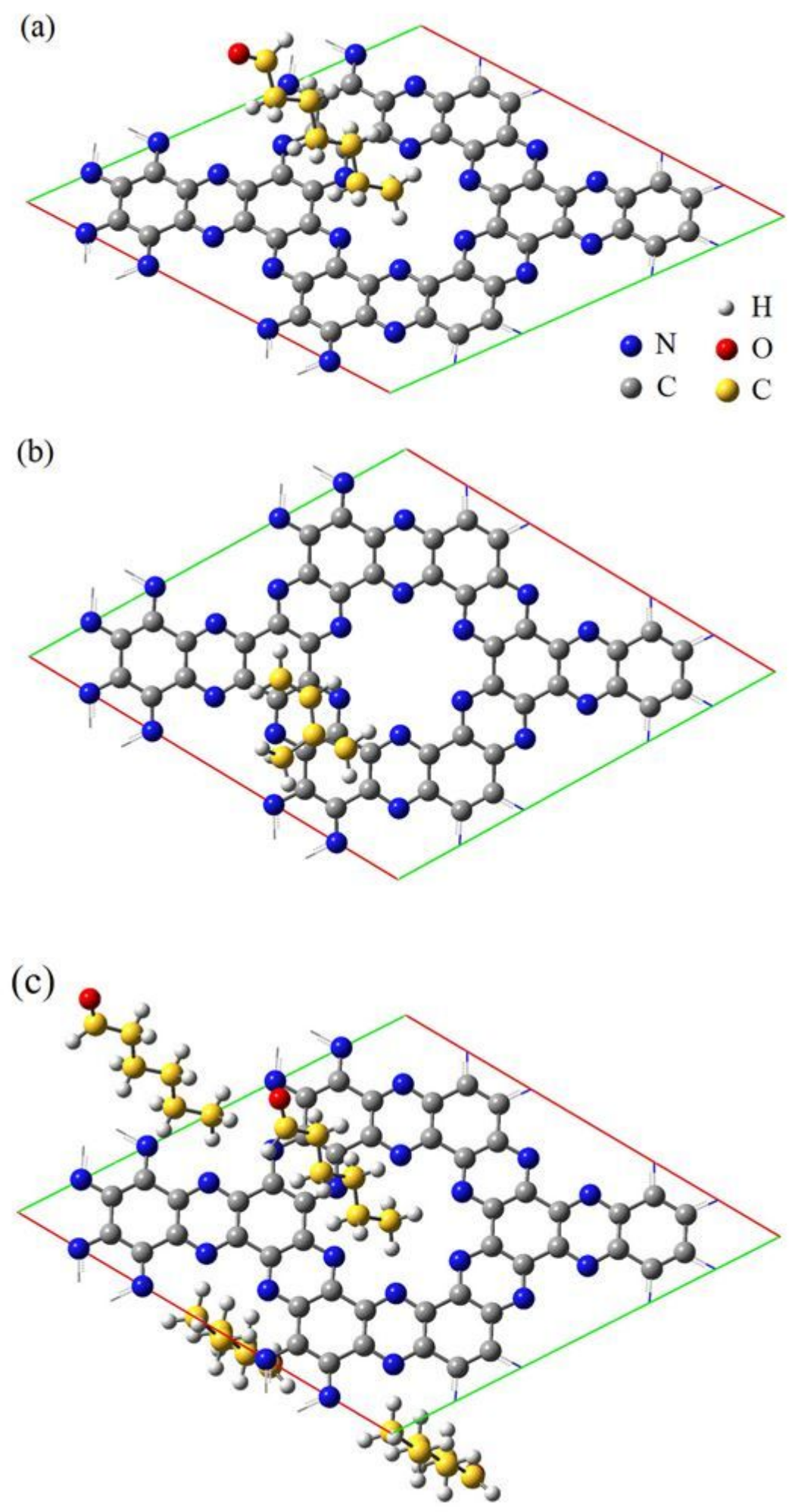

Figure 4

Atomic structures of NHG sheet with adsorbed (a) one heptanal, (b) one isoprene, and (c) four hexanal molecules. 

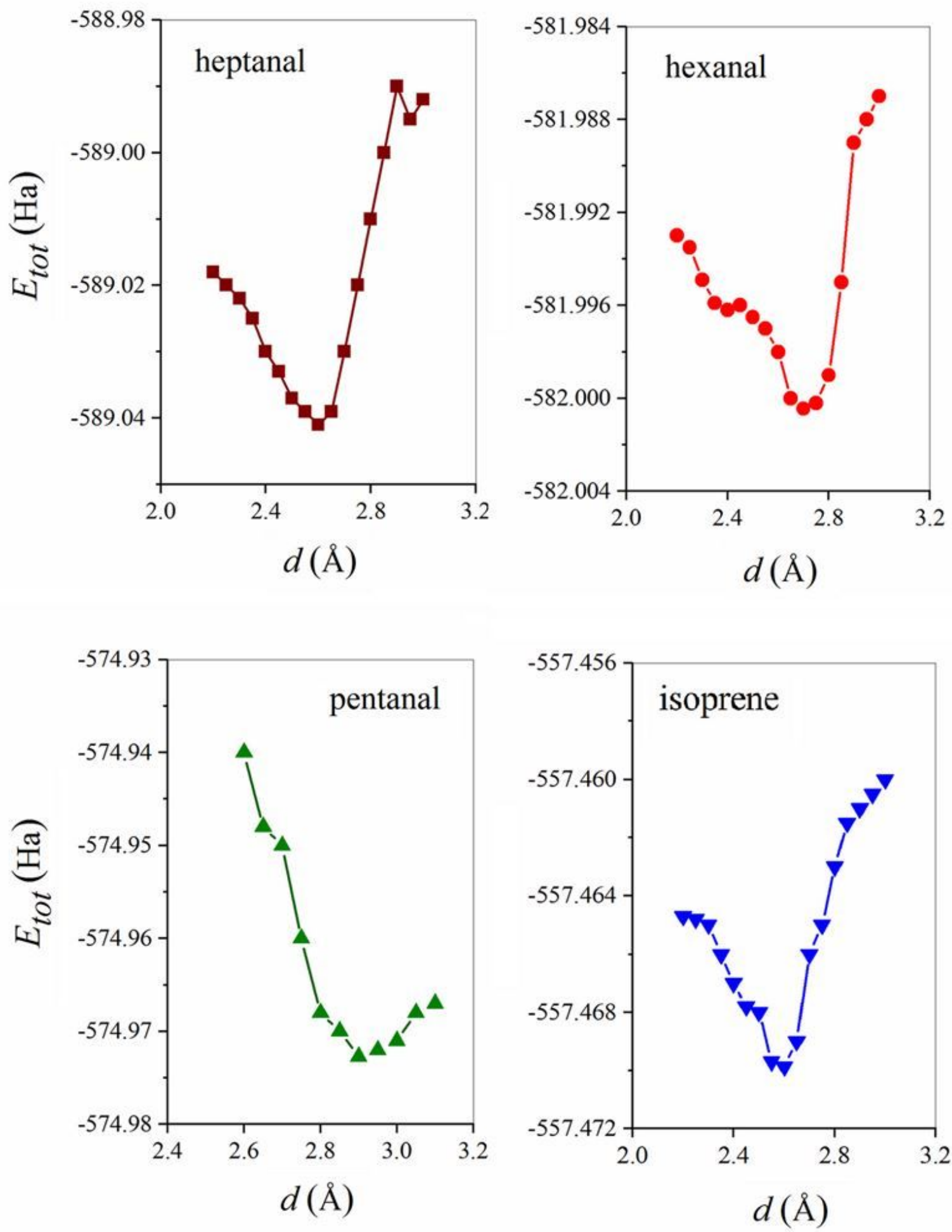

Figure 5

Total energy as a function of adsorption distance taken from NEB calculations. 
(a)
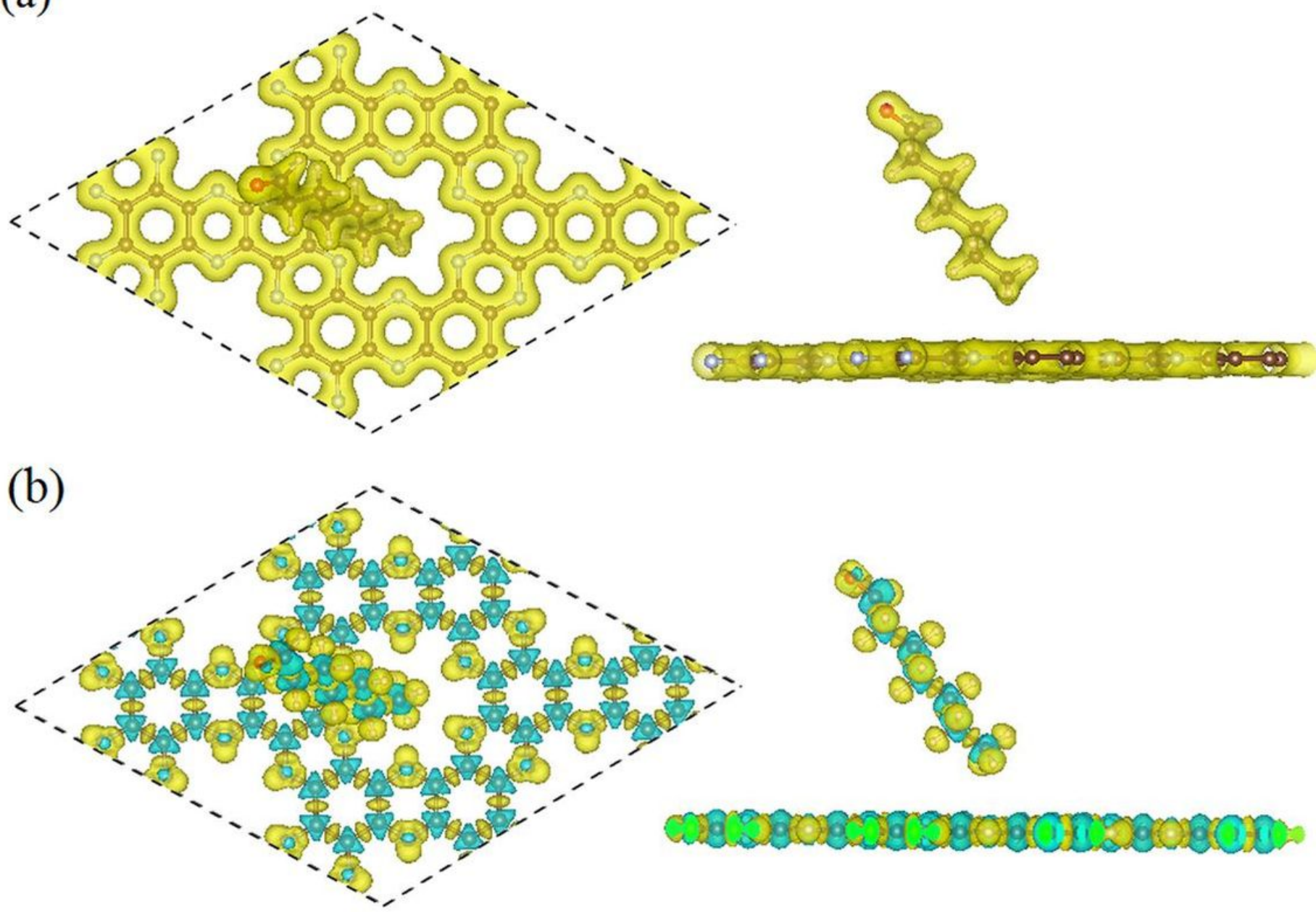

Figure 6

Top and side views of (a) total electron density and (b) electron density difference of NHG sheet with adsorbed heptanal. (The value of isosurface is $0.013 \mathrm{e} / \AA ̊ \AA 3$. The yellow and cyan regions show the gain and loss of electron, respectively.) 

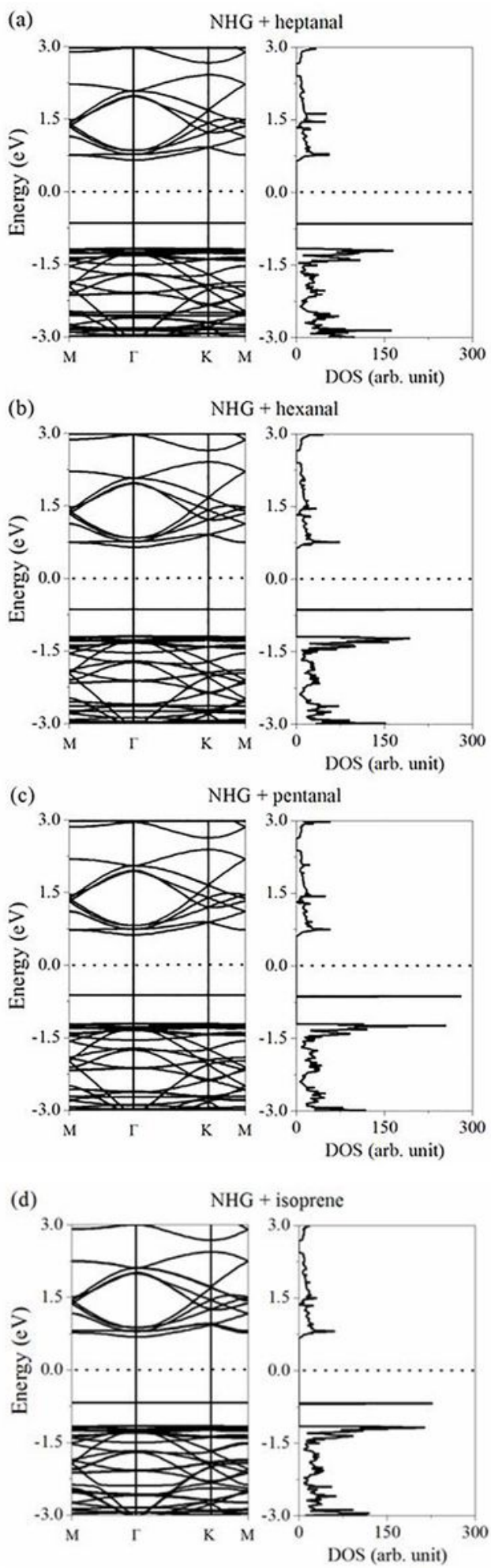

\section{Figure 7}

Electronic band structures and DOS of NHG sheet with adsorbed (a) heptanal, (b) hexanal, (c) pentanal, and (d) isoprene. 


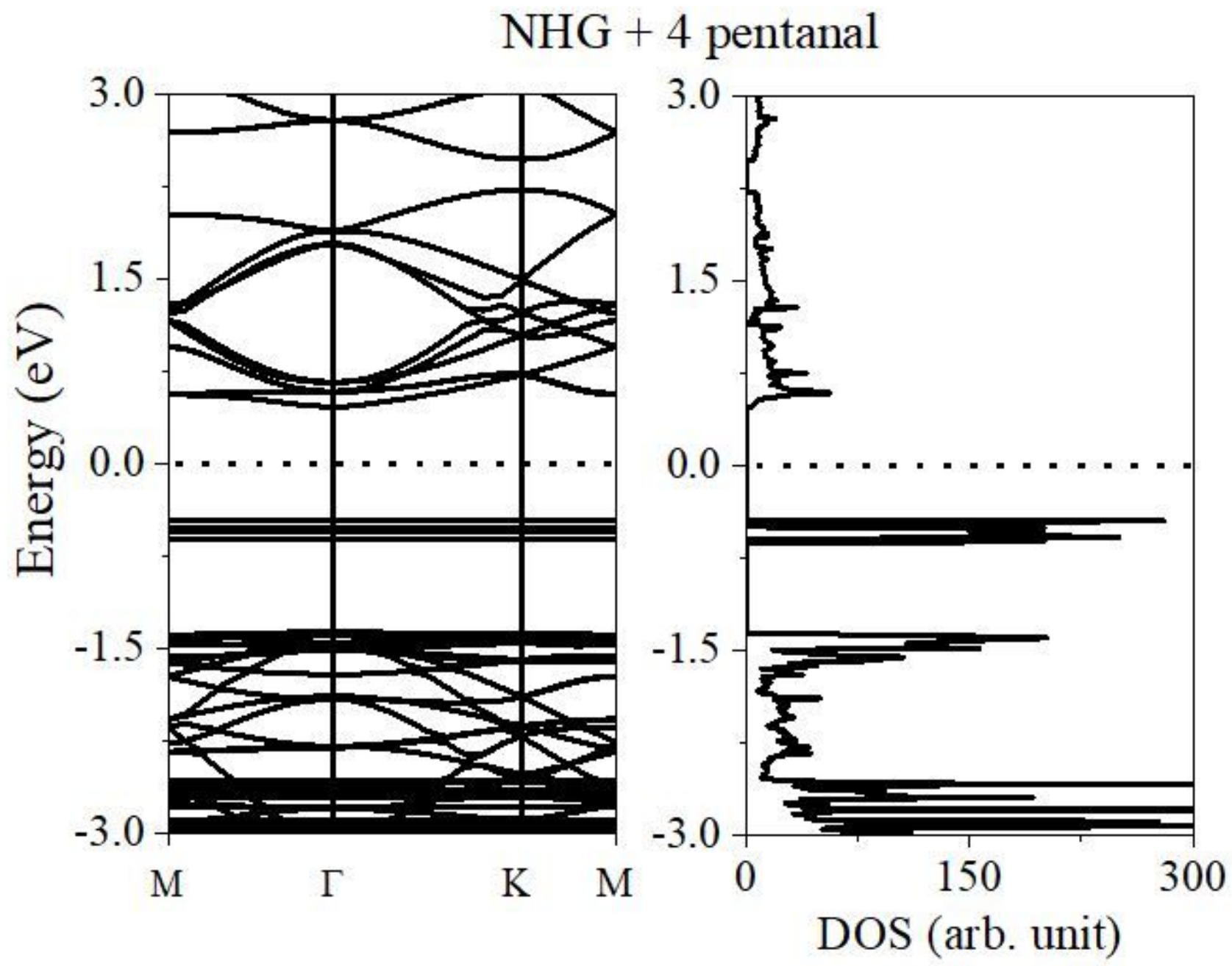

Figure 8

Electronic band structures and DOS of NHG sheet with four adsorbed pentanal molecules. 